\title{
Displacement and re-accumulation of centromeric cohesin during transient pre-anaphase centromere splitting
}

\author{
Maria T. Ocampo-Hafalla • Yuki Katou • \\ Katsuhiko Shirahige $\cdot$ Frank Uhlmann
}

Received: 30 April 2007 / Revised: 5 July 2007 / Accepted: 6 July 2007 / Published online: 1 September 2007

(C) Springer-Verlag 2007

\begin{abstract}
The ring-shaped cohesin complex links sister chromatids until their timely segregation during mitosis. Cohesin is enriched at centromeres where it provides the cohesive counterforce to bipolar tension produced by the mitotic spindle. As a consequence of spindle tension, centromeric sequences transiently split in pre-anaphase cells, in some organisms up to several micrometers. This 'centromere breathing' presents a paradox, how sister sequences separate where cohesin is most enriched. We now show that in the budding yeast Saccharomyces cerevisiae, cohesin binding diminishes over centromeric sequences that split during breathing. We see no evidence for cohesin translocation to surrounding sequences, suggesting that cohesin is removed from centromeres during breathing. Two pools of cohesin can be distinguished. Cohesin loaded before DNA replication, which has established sister chromatid cohesion, disappears during breathing. In contrast, cohesin loaded after DNA replication is partly retained. As sister centromeres re-associate after
\end{abstract}

Communicated by E.A. Nigg

Electronic supplementary material The online version of this article (doi:10.1007/s00412-007-0118-4) contains supplementary material, which is available to authorized users.

M. T. Ocampo-Hafalla $\cdot$ F. Uhlmann $(\bowtie)$

Chromosome Segregation Laboratory,

Cancer Research UK London Research Institute,

44 Lincoln's Inn Fields,

London WC2A 3PX, UK

e-mail: frank.uhlmann@cancer.org.uk

Y. Katou $\cdot$ K. Shirahige

Center for Biological Resources and Informatics,

Division of Gene Research, Tokyo Institute of Technology,

4259 Nagatsuta, Midori-ku,

Yokohama 226-8501, Japan transient separation, cohesin is reloaded in a manner independent of the canonical cohesin loader Scc2/Scc4. Efficient centromere re-association requires the cohesion establishment factor Eco1, suggesting that re-establishment of sister chromatid cohesion contributes to the dynamic behaviour of centromeres in mitosis. These findings provide new insights into cohesin behaviour at centromeres.

\section{Introduction}

Sister chromatid cohesion mediates chromosome stability during cell division. After their synthesis during S-phase, sister chromatids remain physically linked by the chromosomal multi-subunit protein complex cohesin. This allows pairs of replication products to be recognized in mitosis for bipolar alignment on the spindle apparatus. Loss of cohesion at anaphase onset triggers segregation of the sister chromatids towards the nascent daughter cells (reviewed in Uhlmann 2003; Nasmyth and Schleifer 2004).

In the budding yeast Saccharomyces cerevisiae, cohesin consists of at least four essential subunits ( $\mathrm{Smc1}$, Smc3, Scc1, Scc3), which together form a ring-shaped protein complex (reviewed in Nasmyth and Haering 2005; Hirano 2006). The cohesin ring is thought to provide cohesion by topologically embracing sister chromatids (Ivanov and Nasmyth 2005). Loading of cohesin onto chromosomes in budding yeast begins in late G1 when the Scc1 subunit is synthesized and cohesin complexes are assembled. The loading reaction requires a separate complex consisting of the Scc2 and Scc4 subunits (Ciosk et al. 2000). Cohesin is loaded onto chromosomes at the binding sites of the Scc2/ Scc4 complex, at the centromere and at multiple locations along chromosome arms. From these loading sites, cohesin 
translocates to accumulate at regions of convergent transcriptional termination (Lengronne et al. 2004). The largest concentration of cohesin is loaded at centromeres, from where it also appears to spread and to accumulate at surrounding transcriptional termination sites. This establishes a cohesin-enriched region spanning 20-50 kilobases (kb) around the centromere (Blat and Kleckner 1999; Megee et al. 1999; Tanaka et al. 1999; Glynn et al. 2004; Lengronne et al. 2004; Weber et al. 2004). During DNA replication in S-phase, cohesion is established between the newly synthesized sister chromatids in a manner that depends on the acetyltransferase Ecol (Uhlmann and Nasmyth 1998; Skibbens et al. 1999; Tóth et al. 1999). Only cohesin that is present on DNA at the time of DNA replication participates in the establishment of sister chromatid cohesion (Haering et al. 2004; Ström et al. 2004). However, during undisturbed cell cycle progression, cohesin loading appears to be a relatively slow process, and DNA replication initiates and is completed while cohesin continues to associate with chromosomes (Lengronne et al. 2006). This means that cells in metaphase contain two pools of cohesin: one that was loaded before DNA replication and is therefore likely involved in sister chromatid cohesion, and another pool that was loaded only after DNA replication and is therefore not able to promote cohesion. At anaphase onset, all cohesin dissociates from chromosomes after cleavage of the Scc1 subunit by the protease separase (Uhlmann et al. 2000). This abolishes cohesion and releases sister chromatids for segregation into opposite directions.

Cohesin binding sites along chromosome arms are found with an average spacing of approximately $15 \mathrm{~kb}$. The likely task for cohesin along chromosome arms is to keep sister sequences aligned in proximity to facilitate DNA break repair by homologous recombination (Sjögren and Nasmyth 2001). For its role in supporting bi-orientation of sister chromatids on the mitotic spindle, cohesin at the centromere of each chromosome should in principle suffice. Indeed, in metazoan cells, most of cohesin dissociates from chromosome arms as cells enter mitosis (Losada et al. 1998; Waizenegger et al. 2000). This does not require cohesin cleavage by separase and occurs by a mechanism called the prophase pathway of cohesin removal. Only cohesin around centromeres is protected from removal in prophase and is retained to support chromosome biorientation in metaphase (Salic et al. 2004; Kitajima et al. 2005; McGuinness et al. 2005).

These considerations suggest that cohesin at centromeres is of particular importance for chromosome segregation. However, the mechanism underlying the pronounced centromeric accumulation of cohesin in budding yeast is not clear. Assembly of inner and central kinetochore components onto an intact centromere core sequence is required, but how this facilitates cohesin loading remains unknown (Megee et al. 1999; Tanaka et al. 1999; Weber et al. 2004; Eckert et al. 2007). In fission yeast, cohesin is also most abundant at centromeres where its accumulation depends on the heterochromatin protein Swi6. Swi6 associates with the outer centromeric repeat regions, and its recruitment of cohesin probably involves the direct interaction with the Psc3 (pombe Scc3) subunit (Bernard et al. 2001; Nonaka et al. 2002). Depletion of Swi6 leads to a loss of cohesin from centromeres, but not chromosome arms, accompanied by severe chromosome segregation defects. This is consistent with the crucial role of centromeric sister chromatid cohesion in chromosome bi-orientation. Cohesin staining along human, Xenopus and Drosophila mitotic chromosomes has also shown an enrichment of cohesin at centromeres (Losada et al. 2000; Waizenegger et al. 2000; Warren et al. 2000). In these cases, it is not yet known whether a specific mechanism leads to preferential loading of cohesin at centromeres or whether the relative enrichment seen in mitosis is merely the consequence of cohesin removal from chromosome arms.

The accumulation of cohesin suggests that sister chromatid cohesion is particularly strong at centromeres. Firm cohesion might be required to withstand the pulling force of the mitotic spindle that attaches here. This, however, seems to be at odds with observations that centromeric sister sequences split for significant distances during the chromosome bi-orientation process, long before separase is activated to remove cohesin from centromeres. This phenomenon of a "pre-metaphase stretch" was early observed during alignment of sister centromeres on meiosis II spindles in spermatocytes of the grasshopper Isagoras subaquilus (Hughes-Schrader 1950). Since then, it has become clear that centromeric sequences in many species, including human, fission yeast and budding yeast, separate under the tension of the mitotic spindle during prophase and metaphase (Shelby et al. 1996; Nabeshima et al. 1998; Goshima and Yanagida 2000; He et al. 2000; Tanaka et al. 2000; Pearson et al. 2001). This pre-anaphase sister separation is often transient and followed by re-association of the sister centromeres. Therefore, it has been referred to as 'centromere breathing.'

Centromere breathing in budding yeast leads to transient separation of sister centromeres for up to $0.8 \mu \mathrm{m}$ and causes splitting of sequences up to $9 \mathrm{~kb}$ away from the centromere (Goshima and Yanagida 2000; He et al. 2000). Breathing is therefore confined within the larger region of cohesin enrichment surrounding the centromere. A stretched cohesin ring that embraces both sister chromatids at centromeres could span $0.05 \mu \mathrm{m}$ at most, and, consequently, it appears impossible for cohesin to maintain sister chromatid cohesion during centromere breathing. The only known activity that can remove cohesin from mitotic chromosomes in budding 
yeast, the protease separase, is not required for centromere breathing (He et al. 2000; Tanaka et al. 2000). Thus, it has remained unknown how centromere splitting can occur exactly at the place where cohesin is most highly enriched. This paradox has sometimes been used to put into question a role of cohesin in providing physical linkage between sister centromeres.

We have now analysed the behaviour of cohesin at budding yeast centromeres during centromere breathing. We have used chromatin immunoprecipitation, followed by hybridisation to high-density oligonucleotide microarrays to characterise the distribution pattern of cohesin at centromeres during breathing. This has revealed that within the larger region of centromere enrichment, cohesin association is strongly reduced over an approximately $10 \mathrm{~kb}$ core region when centromeres split during breathing. Similar observations have recently been made by Eckert et al. (2007). Furthermore, we find that a pool of cohesin that has been loaded at centromeres after DNA replication, and is therefore not involved in sister chromatid cohesion, is partly retained at centromeres during breathing. This suggests that it is not cohesin's presence at centromeres per se, but rather its involvement in physical sister linkages, which becomes incompatible with centromere breathing. We also show that re-association of sister centromeres after transient splitting correlates with reloading of cohesin and is promoted by the sister chromatid cohesion establishment factor Eco1. These findings resolve the centromere breathing paradox and provide new insight into cohesin behaviour during the establishment of bipolar tension at centromeres.

\section{Materials and methods}

Yeast strains and growth conditions

All $S$. cerevisiae strains used in this study were derivatives of W303. Epitope tagging of endogenous genes was performed by gene targeting using polymerase chain reaction products (Knop et al. 1999). Cells containing the MET3-CDC20 allele were grown at $25^{\circ} \mathrm{C}$ in $\mathrm{SC}$ medium lacking methionine with $2 \%$ raffinose or $2 \%$ glucose as the carbon source (Uhlmann et al. 2000). Cultures were synchronized in G1 with $\alpha$-factor $(5 \mu \mathrm{g} / \mathrm{ml})$ and released into medium containing $100 \mathrm{mM}$ hydroxyurea (HU) for arrest in early S-phase. For arrest in metaphase, cells were released from $\alpha$-factor or $\mathrm{HU}$ arrest by filtration and resuspension in YP medium supplemented with $5 \mathrm{mM}$ methionine to deplete Cdc20. To inactivate cohesin loading, cells harbouring the temperature sensitive $s c c 2-4$ allele (Ciosk et al. 2000) were shifted to the restrictive temperature of $35^{\circ} \mathrm{C}$ for $1 \mathrm{~h}$ before release. To prevent metaphase spindle assembly or to disassemble already formed spindles, $7.5 \mu \mathrm{g} / \mathrm{ml}$ nocodazole was added to the medium for $1 \mathrm{~h}$. To wash out nocodazole, cells were filtered again, washed and resuspended in fresh medium lacking nocodazole for $1 \mathrm{~h}$. Expression of Scc1 to induce differentially epitope-tagged cohesin under control of the GAL1 promoter, or of Cdc14, was achieved by addition of $2 \%$ galactose for $1 \mathrm{~h}$ to cultures grown in raffinose-containing medium. In all experiments, synchrony of cell cycle progression was confirmed by flow cytometry of DNA content.

\section{Chromatin immunoprecipitation}

Cohesin distribution along budding yeast chromosomes was analysed by chromatin immunoprecipitation against epitope-tagged Scc1 as previously described (Katou et al. 2003; Lengronne et al. 2004). Chromatin immunoprecipitates were hybridised to Affymetrix $S$. cerevisiae chromosome 6 (rikDACF; P/N 510636) or whole genome forward tiling arrays (P/N 520286). Details of the chromatin immunoprecipitation experiments and the microarray data presented in this article can be obtained from Gene Expression Omnibus with accession number GSE 8661.

\section{Microscopy}

GFP marked centromeres were observed in $100 \%$ ethanol fixed samples. Mitotic spindles were visualised by indirect immunofluorescence in formaldehyde-fixed cells using $\alpha$ tubulin antibody YOL1/34 (Serotec). To assess centromere splitting at each time point, $\geq 100$ cells were scored for the presence of one or two GFP signals using an Axioplan 2 imaging microscope (Zeiss) equipped with a $100 \times(\mathrm{NA}=$ 1.45) Plan-Neofluar objective.

\section{Results}

Reduced cohesin levels at breathing centromeres

To study the relationship between centromere breathing and cohesin, we analysed mitotic cohesin distribution around the centromere of budding yeast chromosome 6 in the presence or absence of mitotic spindle forces that drive breathing. Cells were released from $\alpha$-factor block in G1 into synchronous cell cycle progression and arrested in metaphase by depletion of the anaphase-promoting complex co-activator Cdc20 under control of the repressible MET3 promoter. To half of the culture, the microtubule poison nocodazole was added at the time of G1 release to inhibit spindle formation. Cohesin localisation was then analysed in both cultures by chromatin immunoprecipitation against an HA epitope tag attached to the Scc1 subunit followed by hybridisation to an oligonucleotide microarray 
covering chromosome 6 . The Scc1 pattern in the presence of nocodazole was similar to what has been previously observed under these conditions, including a strong enrichment around the centromere (Lengronne et al. 2004). The highest peak of association coincided with the core centromere sequence (Fig. 1). Metaphase-arrested cells in the absence of nocodazole showed a strikingly different pattern. The overall enrichment of Scc1 around the centromere was still apparent, but the core centromere sequence was clear of Scc1, and its levels within the surrounding several kilobases were significantly reduced. The Scc1 pattern further away from the centromere, and along the chromosome arms, was unchanged. To confirm that the Scc1 pattern accurately reflected the localisation of the cohesin complex, we also assessed the distribution of the Smc3 subunit under the same conditions. Like Scc1, Smc3 association in the presence of nocodazole was most pronounced around the centromere (Electronic supplementary Fig. 1). Also consistent with the distribution of Scc1, Smc3 accumulation at the core centromere sequence was greatly diminished in the absence of nocodazole, while the pattern along chromosome arms remained unchanged. This demonstrates that cells, which enter mitosis in the presence or absence of spindle microtubules and therefore do or do not undergo centromere breathing, show a strikingly different cohesin pattern at the centromere.
Cohesin removal during centromere splitting

We next wanted to address whether reduced centromeric cohesin was due to cohesin removal in response to centromere splitting. For this, we first arrested cells in metaphase in the presence of nocodazole and then washed out the microtubule drug to initiate spindle assembly and centromere breathing, while cells were maintained in the metaphase arrest. To focus our analysis on cohesin that has established sister chromatid cohesion during S-phase, we utilised an experimental scheme to prevent continued cohesin loading onto chromosomes after DNA replication (Lengronne et al. 2006). After release from G1, cells were first arrested with the replication inhibitor HU. At this stage, cohesin is loaded onto chromosomes, but the centromere of chromosome 6 has not been replicated (Katou et al. 2003). Further cohesin loading was then turned off by inactivating the temperature sensitive cohesin loader allele scc2-4 before releasing cells to progress through S-phase and into metaphase arrest in the presence of nocodazole (Fig. 2, state a). The conformation of centromeres in the arrest was followed by visualisation of a GFP marked locus $1.4 \mathrm{~kb}$ from the centromere of chromosome 5 (Tanaka et al. 2000). Ninety-three percent of cells exhibited single GFP dots in the nocodazole arrest, confirming that centromere breathing was inhibited in the
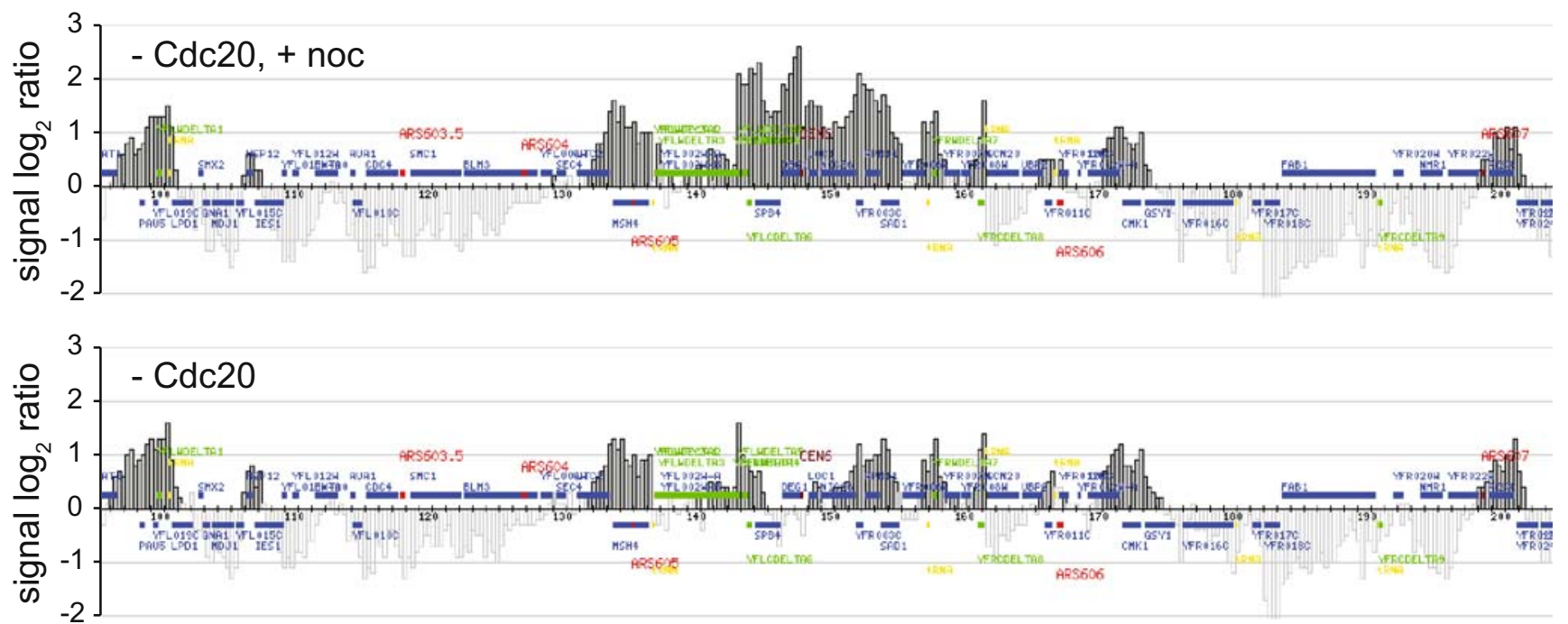

Fig. 1 Prominent centromeric cohesin enrichment in the absence of spindle forces. Cells of strain Y379 (MATa MET3-CDC20 SCC1-HAO) were synchronized in G1 with $\alpha$-factor and released into metaphase arrest by $\mathrm{Cdc} 20$ depletion in the presence $(-\mathrm{Cdc} 20+$ noc) or absence of $(-\mathrm{Cdc} 20)$ nocodazole. Cultures were processed for chromatin immunoprecipitation against the HA epitope-tagged cohesin subunit Scc1. Enrichment of DNA fragments in the immunoprecipitate relative to a whole genome sample is shown over an approximately $110 \mathrm{~kb}$-wide region surrounding centromere 6 . Each bar represents the average of
16 oligonucleotide probes within adjacent $300 \mathrm{bp}$ windows. The $y$ axis scale is $\log _{2}$. Grey signals represent significant binding as described (Katou et al. 2003). Blue bars above and below the midline are genes transcribed from left to right and opposite, respectively. The centromere is depicted in dark red, origins of replication are in red, tRNA genes in yellow and Ty elements in green. The cohesin signal over Ty elements is not interpretable in this analysis due to their repetitive nature in the genome 
presence of nocodazole. The cohesin pattern at centromere 6 was very similar to what was observed above in Fig. 1. We then washed out nocodazole, which, within $1 \mathrm{~h}$, led to formation of metaphase spindles (data not shown) and to splitting of the centromeric GFP signal in $77 \%$ of cells, as expected during centromere breathing. At the same time, cohesin disappeared from centromere 6 and several kilobases of flanking sequence (Fig. 2, state b). To the right of the centromere, only little cohesin remained over a region of almost $10 \mathrm{~kb}$. This corresponds approximately to the region
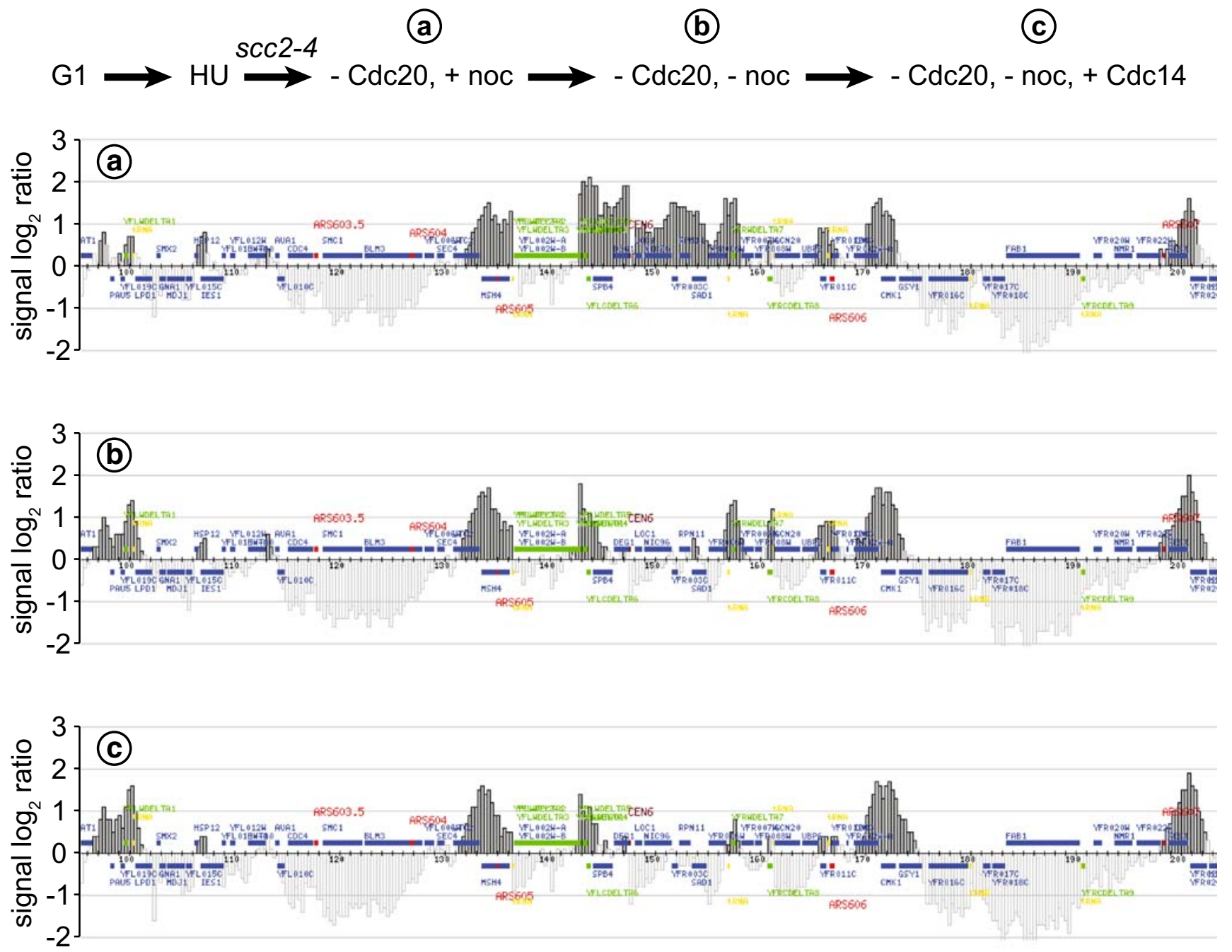

(a)

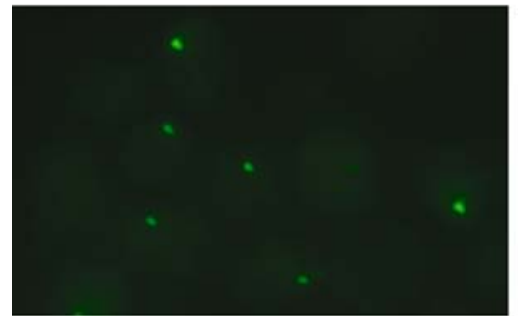

Fig. 2 Cohesin removal from centromere 6 during pre-anaphase splitting. Cells of strain Y3138 (MATa scc2-4 MET3-CDC20 SCC1-HA6 GAL1CDC14 CEN5(1.4 kb)tetOs tetR-GFP) were synchronized in G1 with $\alpha$ factor and released into $\mathrm{HU}$ arrest in early S-phase before Scc2 was inactivated by temperature shift. Cells were released into metaphase arrest (b)

(c)
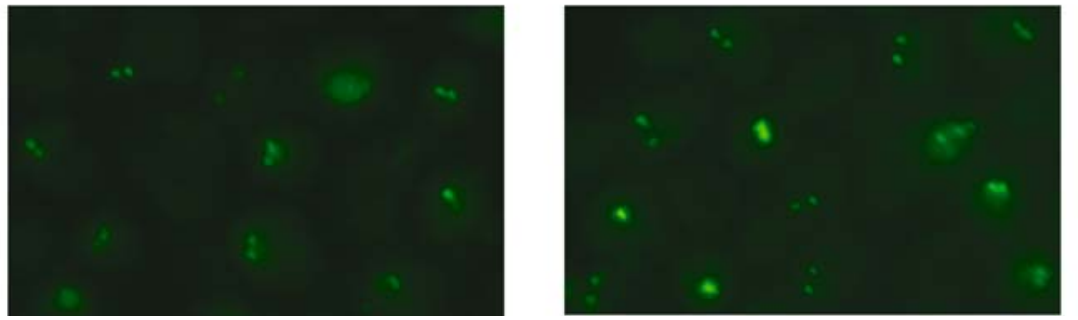

by $\mathrm{Cdc} 20$ depletion in the presence of nocodazole (state $\boldsymbol{a}$ ). Nocodazole was then washed out to allow spindle assembly and centromere splitting (state $\boldsymbol{b}$ ), and $\mathrm{Cdc} 14$ expression was induced by galactose addition to stabilise split centromeres (state $\boldsymbol{c}$ ). Cohesin distribution around centromere 6 and splitting of the GFP-marked centromere 5 were analysed 
expected to separate during centromere breathing (He et al. 2000). We did not see evidence for a compensatory increase of cohesin outside the depleted region, as would be expected if cohesin was laterally displaced during centromere splitting. This suggests that cohesin is lost from centromeres. The levels of remaining centromeric cohesin in this experiment were strikingly lower when compared to metaphase arrested cells in Fig. 1. A possible explanation for this difference is that in the previous experiment, cohesin at centromeres was a combination of complexes loaded before as well as after DNA replication, and that these two pools of cohesin may behave differently during centromere breathing. This possibility is further considered below.

Loss of cohesin from centromeres after nocodazole washout suggested that it was the consequence of spindle assembly and of initiation of centromere breathing. Forceful splitting of sister centromeres by bipolar spindle tension may disrupt cohesin's contact with DNA. Alternatively, loss of cohesin could be an indirect consequence of removing the spindle poison, e.g. cohesin could respond to the spindle checkpoint signal that is activated in the presence of nocodazole. To distinguish between these possibilities, we asked whether a correlation existed between the extent of physical sister chromatid splitting and the decrease of cohesin at the centromere. Activation of the Cdc14 phosphatase, which normally occurs at anaphase onset, promotes movement of kinetochores towards the spindle poles (Higuchi and Uhlmann 2005). Ectopic Cdc14 expression in metaphase is sufficient to induce a precocious anaphase A-like kinetochore movement, thereby bringing centromeres into a stably split configuration. Therefore, we overexpressed Cdc14 in the metaphase-arrested cells from above. As a consequence, the percentage of cells with split centromere 5 signals rose to $86 \%$, and the average distance between the signals increased. At the same time, cohesin levels at centromere 6 further decreased (Fig. 2, state c). Ten kilobases to the right of the centromere were now completely free of cohesin, and the signal as far as $20 \mathrm{~kb}$ away was reduced. Cohesin association to the left of centromere 6 was more difficult to assess because of the presence of a Ty 2 retrotransposon and long terminal repeat elements of several additional transposons. Their repetitive presence in the yeast genome makes examination of cohesin association with an individual copy impossible using our technique. From these observations, we conclude that the loss of cohesin from centromeres correlates with their extent of splitting. We cannot exclude the formal possibility that ectopic $\mathrm{Cdc} 14$ expression affects cohesin distribution in another indirect manner. It has recently also been demonstrated that kinetochore mutants that weaken spindle attachment reduce the extent of cohesin loss and that a functional spindle checkpoint is not required to promote cohesin enrichment in the presence of nocodazole
(Eckert et al. 2007). Together, these results suggest that cohesin is removed from regions that split during centromere breathing most likely as the consequence of their physical separation.

We next asked whether removal of cohesin in response to centromere splitting could be observed at the centromere not only of chromosome 6 but also of other budding yeast chromosomes. Therefore, we repeated the analysis shown in Fig. 2, but hybridised the chromatin immunoprecipitates to microarrays containing oligonucleotide probes covering all of the budding yeast genome. Figure 3 compares the cohesin patterns at the 16 budding yeast centromeres either in the absence of breathing (+nocodazole) or with centromeres in permanently split conformation after removal of the drug and expression of Cdc14 (-nocodazole, $+\mathrm{Cdc} 14)$. Cohesin was enriched at all centromeres in the presence of nocodazole and markedly reduced around the core centromere sequence in response to centromere splitting. The response of cohesin to centromere splitting varied between chromosomes, from complete abrogation of binding (e.g. chromosome 1) to a reduction of cohesin without change to the overall association pattern (e.g. chromosome 3). In many cases, distinct boundaries existed between regions of cohesin loss and sequences where cohesin levels remained unaffected. The boundaries on both sides of the centromere were not always symmetric (e.g. chromosome 7). This suggests that centromere splitting causes removal of at least part of cohesin from all budding yeast centromeres. Unlike centromere 6 , centromeres of most other chromosomes replicate early in S-phase and are, at least partly, replicated in an HU-imposed S-phase block (Y. K. and K. S., unpublished observations). Therefore, a mixture of pre- and post-replicatively loaded cohesin was likely present at most centromeres. This might explain the persistence of some cohesin at these centromeres during breathing.

Differential behaviour of pre- and post-replicative loaded cohesin during centromere breathing

Loading of cohesin onto chromosomes begins in late G1, when the Scc1 subunit is synthesised, and continues throughout S-phase and G2 (Michaelis et al. 1997; Lengronne et al. 2006). Cohesion between sister chromatids is established during DNA replication, involving only those cohesin complexes that have already bound to chromosomes at that time. A possible function for cohesin that is still loaded onto chromosomes after DNA replication is not known, but it does not promote sister chromatid cohesion under unchallenged conditions (Haering et al. 2004; Ström et al. 2004; Lengronne et al. 2006). If removal from centromeres during breathing is the consequence of physical splitting of DNA molecules that are held together by cohesin, only cohesin complexes that are involved in sister 
(a) + noc $\quad$ (c) - noc, $+\mathrm{Cdc} 14$
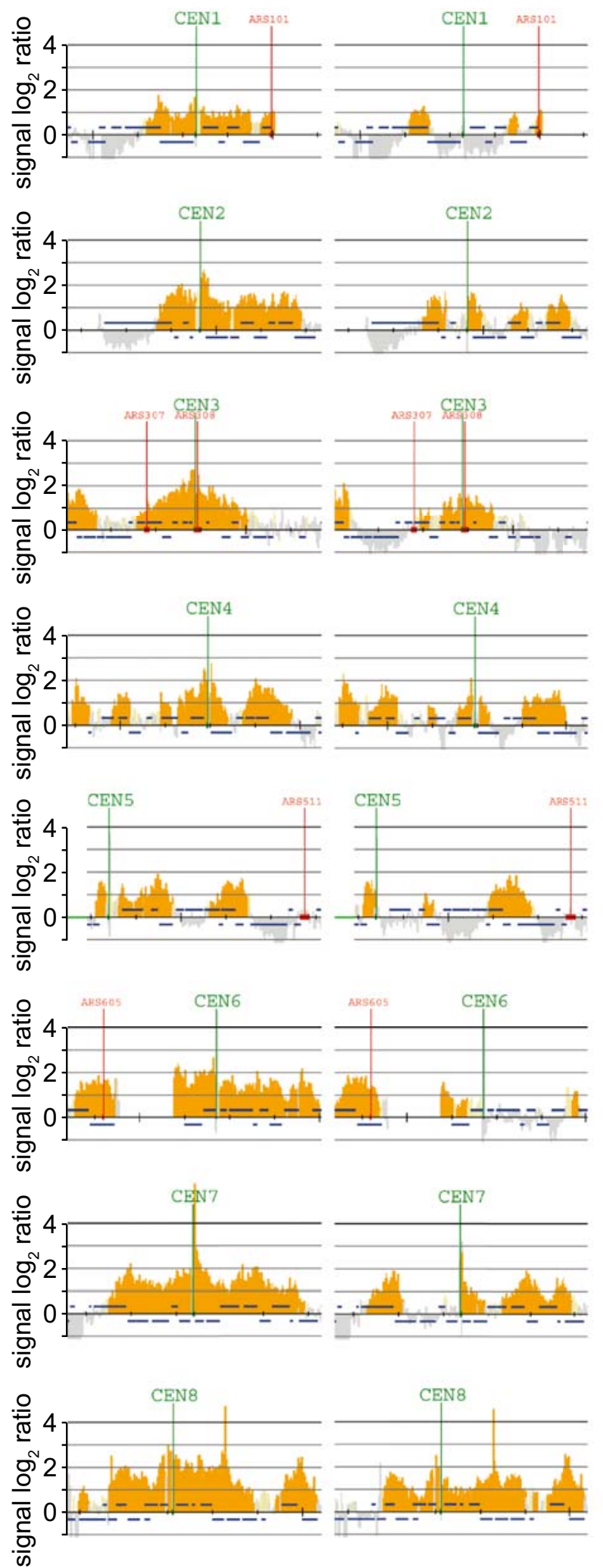

Fig. 3 Cohesin removal from all centromeres in response to centromere splitting. As in Fig. 2, but the cohesin immunoprecipitate was hybridised to a microarray covering all of the budding yeast genome. States $\boldsymbol{a}$, no centromere breathing in the presence of nocodazole and $\boldsymbol{c}$, permanently split centromeres after nocodazole washout and Cdc14
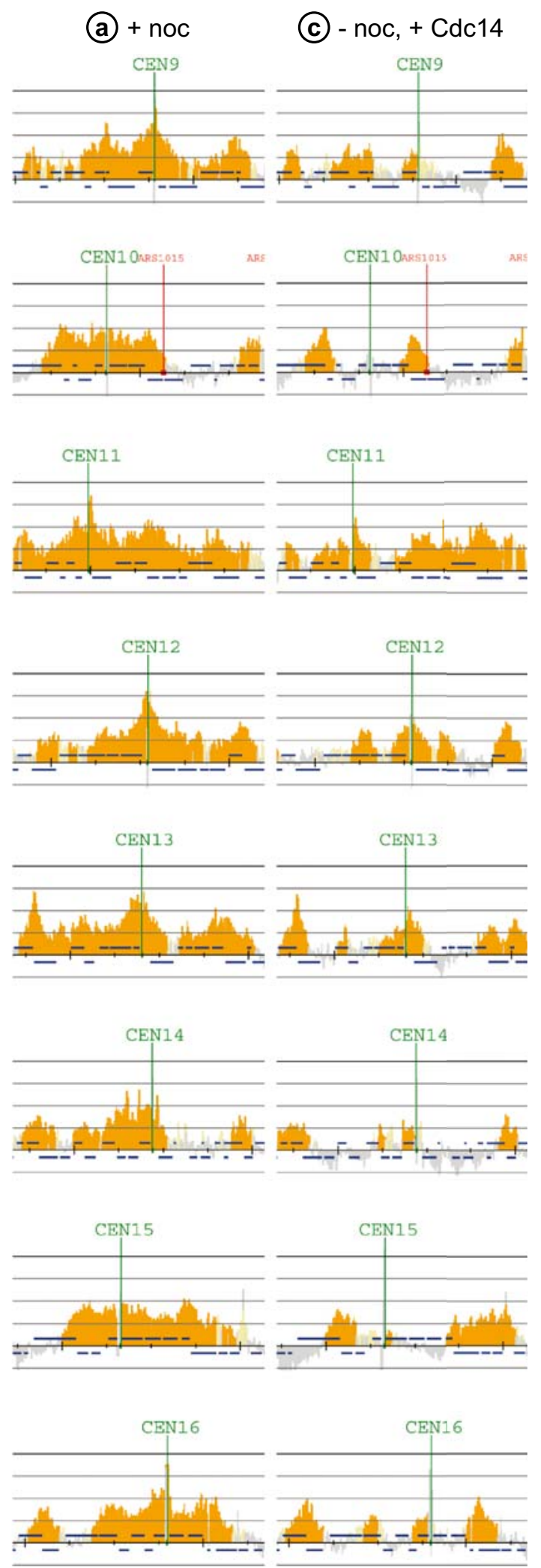

induction are presented. Cohesin distribution over a region of approximately $30 \mathrm{~kb}$ surrounding each centromere is shown. Tick marks along the $x$-axis represent $5 \mathrm{~kb}$ intervals. Origins of replication are indicated at a selection of chromosomes. The green line to the left of centromere 5 indicates the location of the tetO repeats 
chromatid cohesion should be dislodged. That is, cohesin that does not promote sister chromatid cohesion may not be forced to dissociate. Circumstantial evidence from our experiments above indeed suggested that non-cohesive cohesin loaded after DNA replication may not dissociate from centromeres during breathing. The extent of cohesin removal from centromere 6 was greater in cells where postreplicative loading was prevented (compare Figs. 1 and 2).

To test more directly whether pre- and post-replicatively loaded cohesin behaved differently during centromere breathing, we employed cells in which additional cohesin could be added at specified times by induction of HA epitope-tagged Scc1 under control of the galactose-inducible GAL1 promoter. Cells were synchronised in G1 by $\alpha$-factor treatment and released into HU-imposed arrest in early Sphase. In half of the culture, Scc1-HA was now induced by addition of galactose (HU-loaded). After $1 \mathrm{~h}$, induction was terminated by shifting cells to glucose-containing medium. Next, both cultures were released from the HU block to pass through S-phase and arrested again in mitosis in the presence of nocodazole. Then, Scc1-HA was induced for $1 \mathrm{~h}$ in the other culture (noc-loaded) before induction was terminated by shifting to glucose. Chromatin immunoprecipitation analysis of the HU- or noc-loaded cohesin showed the expected enrichment of both pools around the centromere of chromosome 6 (Fig. 4). The enrichment of cohesin at centromeres, vs chromosome arms, was more pronounced in the case of noc-loaded cohesin. This suggests that a pathway for preferential centromeric cohesin-loading might be particularly active in mitotic cells. We then washed out nocodazole from both cultures to allow spindle assembly and centromere breathing, while cells remained arrested in metaphase due to $\mathrm{Cdc} 20$ repression. This led to the complete removal of HU-loaded cohesin from centromeres (Fig. 4). In contrast, noc-loaded cohesin was only partly removed, and significant binding at the centromere was maintained. This confirms the notion that cohesin loaded before DNA replication is preferentially removed during centromere breathing. This pool of cohesin is likely involved in sister chromatid cohesion, and its presence at centromeres may be incompatible with centromere breathing. In contrast, nocloaded cohesin is unable to participate in sister chromatid cohesion, and its presence at centromeres may not interfere with centromere splitting. While noc-loaded cohesin remained at the centromere, its level was also reduced in response to centromere breathing. This suggests that even post-replicatively loaded cohesin responds to centromere splitting. This could be because removal of cohesin is accomplished by a pathway that is not solely sensitive to physical hindrance of centromere splitting. Alternatively, post-replicatively loaded cohesin might gain some cohesiveness at centromeres and therefore respond, in part, similarly to pre-replicatively loaded cohesin (see below).
Re-establishment of sister chromatid cohesion during centromere re-association

Centromeres often transiently re-associate during preanaphase breathing. The nature of the underlying counterforce that reverses splitting by the mitotic spindle is unknown. It has been observed that centromeric chromatin undergoes elastic stretching during breathing ( $\mathrm{He}$ et al. 2000), and chromatin re-compaction could promote sister centromere re-association. Our observation that cohesin is lost from centromeres during splitting raises the question whether cohesin re-accumulates and indeed whether reestablishment of sister chromatid cohesion participates in centromere re-association. We first addressed whether cohesin re-associates with centromeres when their splitting is reversed. We again started with a synchronous cell culture arrested in early S-phase by HU treatment. Scc2/4 was inactivated by the temperature sensitive $s c c 2-4$ allele before cells were released into a metaphase arrest imposed by Cdc20 depletion. Thus, only cohesin loaded before DNA replication was present at centromere 6 . In the metaphase arrest, GFP-marked centromere 5 proximal sequences were split in $85 \%$ of cells as expected. Centromere 6 in these cells was mostly free of cohesin (Fig. 5a). Centromere reassociation occurs only transiently during centromere breathing (He et al. 2000; Tanaka et al. 2000; Goshima and Yanagida 2001; Pearson et al. 2001) so that its effect on cohesin localisation is difficult to discern within a cell population with mostly split centromeres. To enhance centromere re-association, we therefore added nocodazole to the culture. This led to efficient rejoining of centromere 5 with only $7 \%$ split centromere 5 signals remaining after $1 \mathrm{~h}$. Similar centromere re-association in response to nocodazole has been observed on chromosome 15 (Goshima and Yanagida 2000) and is therefore likely to occur at all centromeres. At the same time, we observed cohesin re-accumulation at centromere 6 (Fig. 5a), resulting in a distribution indistinguishable from that observed before breathing (compare Fig. 2). This suggests that cohesin is reloaded at the centromeres as they re-associate. Note that both cohesin reloading at the centromere and centromere re-association occurred in an apparently Scc2-independent manner. The scc24 allele remained inactivated in metaphase at its restrictive temperature throughout this experiment.

The re-accumulation of cohesin at centromeres at the time of sister centromere re-association opened the possibility that sister chromatid cohesion might be re-established. Establishment of sister chromatid cohesion has primarily been studied in the context of DNA replication during Sphase when it depends on the cohesion establishment factor Eco1 (Skibbens et al. 1999; Tóth et al. 1999). In G2 phase, re-enforcement of sister chromatid cohesion by newly loaded cohesin has been observed at sites of double- 

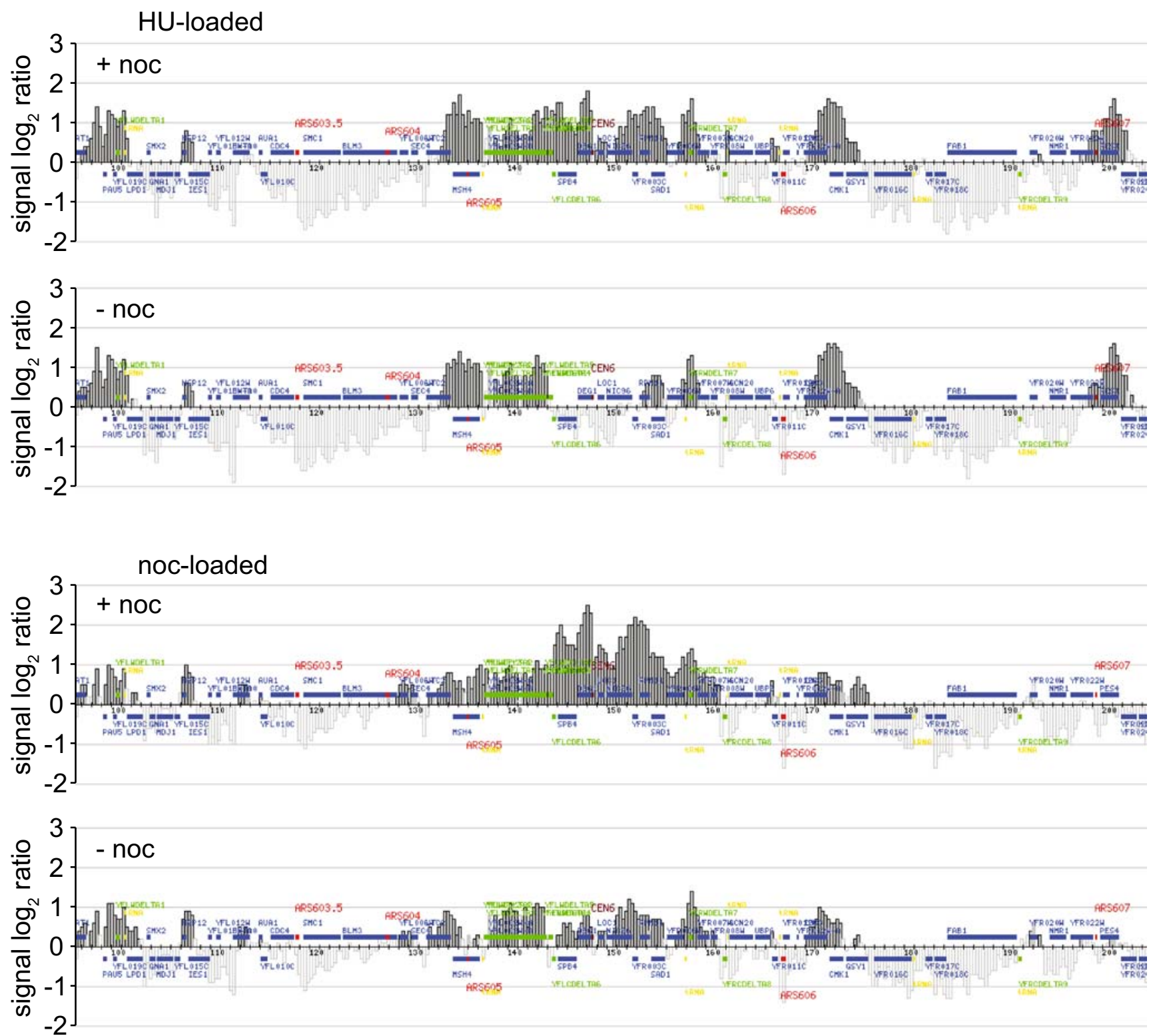

Fig. 4 Differential behaviour of pre- and post-replicatively loaded cohesin. Cells of strain Y3267 (MATa MET3-CDC20 SCC1-Pk9 GAL1-SCC1) were synchronized in G1 with $\alpha$-factor and released into $\mathrm{HU}$ arrest in early S-phase. From there cells were released into metaphase arrest by $\mathrm{Cdc} 20$ depletion in the presence of nocodazole. Loading of ectopic, HA epitope-tagged cohesin was induced by

stranded DNA breaks (Ström et al. 2004). The loss and reaccumulation of cohesin during transient centromere splitting in mitosis suggested that it may be coupled to loss and re-establishment of sister chromatid cohesion. To test whether this was the case, we asked whether Ecol was required for centromere re-association after splitting. We arrested cells carrying the temperature sensitive ecol-1 mutation in metaphase by $\mathrm{Cdc} 20$ depletion. Cells were allowed to enter the arrest at the permissive temperature so that sister chromatid cohesion was established during Sphase. Then Ecol was inactivated by shift to the restrictive induction of the GAL1 promoter for $1 \mathrm{~h}$ either during the time in the HU arrest (HU-loaded), or metaphase arrest (noc-loaded). Nocodazole was then washed out to allow spindle assembly and centromere breathing. Cohesin association around centromere 6 is shown before $(+$ noc $)$ and after nocodazole wash out $(-$ noc $)$

temperature of $35^{\circ} \mathrm{C}$ for $1 \mathrm{~h}$ before nocodazole was added to the culture to eliminate spindle forces. We then monitored whether the GFP-marked centromere 5 was able to re-associate in the absence of Ecol function. In a wildtype control culture treated in the same way, split centromeres were seen in $86 \%$ of metaphase arrested cells. Within $1 \mathrm{~h}$ of nocodazole addition, split GFP signals persisted in only $14 \%$ of cells (Fig. $5 b$ ). Metaphase-arrested eco1-1 cells showed a similar degree of split centromeres $(80 \%)$, but after $1 \mathrm{~h}$ of nocodazole addition, less than half of these re-associated, and centromeres remained separated 
a
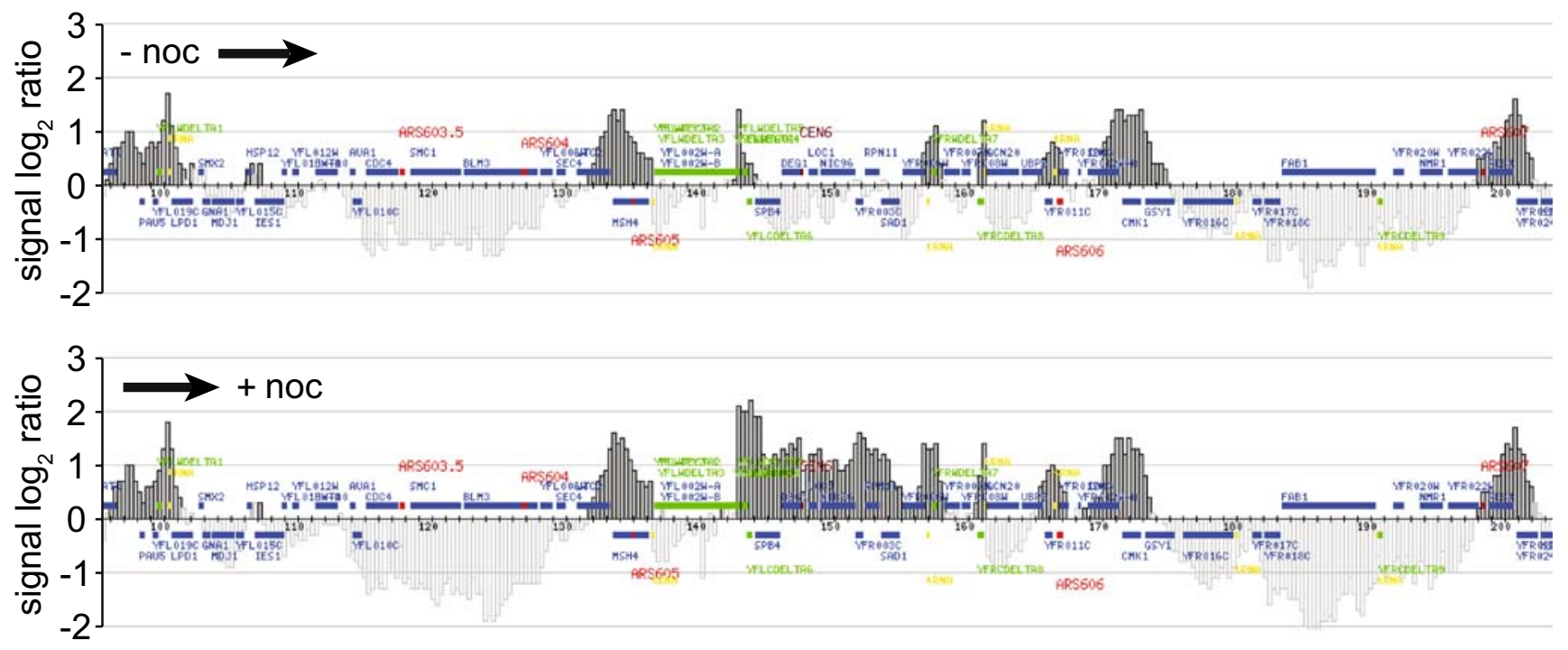

b

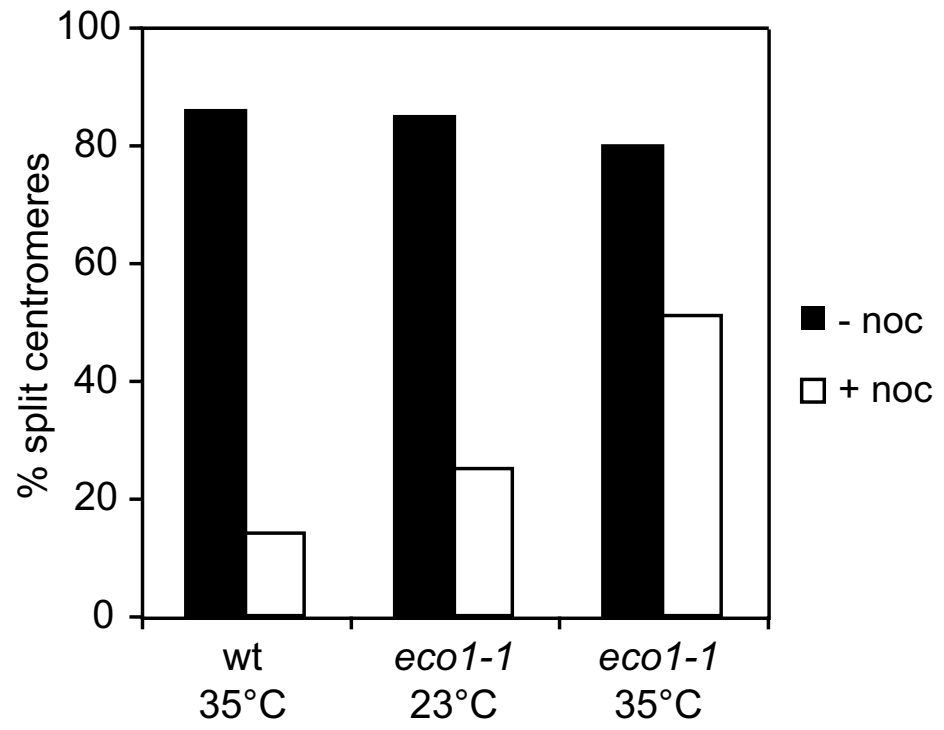

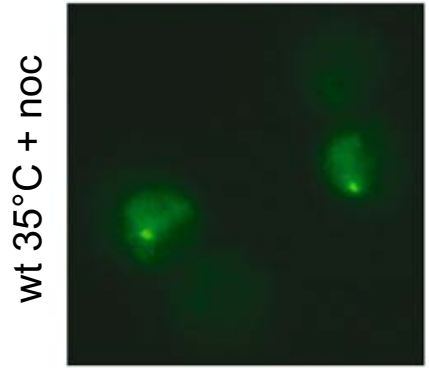

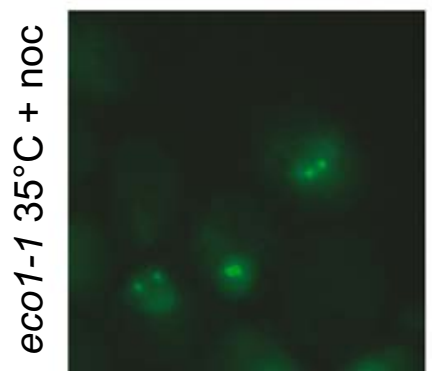

Fig. 5 a Scc2-independent cohesin re-accumulation during sister centromere re-association. Cells of strain Y3138 (MATa scc2-4 MET3CDC20 SCC1-HA6 GAL1-CDC14 CEN5(1.4 kb)tetOs tetR-GFP) were synchronized in G1 with $\alpha$-factor and released into HU arrest in early S-phase before Scc2 was inactivated by temperature shift. From there, cells were released into metaphase arrest by $\mathrm{Cdc} 20$ depletion. Centromere breathing was then stopped and centromere reassociation facilitated by addition of nocodazole. Cohesin distribution around chromosome 6 and splitting of the GFP-marked centromere 5 were analysed before and after nocodazole addition. b Eco1, and therefore most likely re-establishment of sister chromatid cohesion,

in $51 \%$ of cells. Overall, sister chromatid cohesion in mitotic eco1-1 cells is likely compromised even in cells that underwent S-phase at the permissive temperature. It could therefore be that reduced sister centromere re-association observed in this experiment was due to defective cohesion establishment in S-phase rather than to the requirement of Ecol in mitosis. To test this, we added nocodazole to contributes to centromere re-association. Cells of strain Y763 (MATa MET3-CDC20 SCC1-HA6 CEN5(1.4 kb)tetOs tetR-GFP) and Y762 (MATa ecol-1 MET3-CDC20 SCC1-HA6 CEN5(1.4 kb)tetOs tetRGFP) were synchronized in metaphase by $\mathrm{Cdc} 20$ depletion at permissive temperature. Ecol was either inactivated by temperature shift to $35^{\circ} \mathrm{C}$ or retained at permissive temperature for $1 \mathrm{~h}$ before addition of nocodazole to the cultures. Efficient disruption of the spindle by nocodazole in all cells was confirmed by immunostaining (not shown), and splitting of the GFP-marked centromeres was analysed before and $1 \mathrm{~h}$ after nocodozole addition

mitotic ecol-1 cells that were maintained at the permissive temperature. Sister centromere re-association was somewhat compromised, and $25 \%$ of cells retained separated GFP signals out of $85 \%$ split centromeres before nocodazole addition (Fig. 5b). This re-association defect was significantly weaker than observed after mitotic inactivation of the eco1-1 allele. Therefore Eco1, and most likely 
re-establishment of sister chromatid cohesion, contributes to efficient re-association of centromeres in mitosis.

\section{Discussion}

The centromere breathing paradox

Cohesin is found enriched around centromeres of mitotic chromosomes in all species so far studied. There, it plays a crucial role in promoting bi-orientation of sister centromeres by resisting spindle forces during the establishment of tension-based, stable kinetochore-microtubule interactions. In fission yeast, loss of centromeric cohesin due to Swi6 deletion leads to chromosome segregation errors that are likely caused by bi-orientation defects, exemplifying the importance of centromeric cohesin (Bernard et al. 2001; Nonaka et al. 2002). Recently, a budding yeast centromere was modified by insertion of foreign sequences that prevented cohesin accumulation. This also caused chromosome instability, consistent with a crucial role for centromeric cohesin (Eckert et al. 2007). Despite cohesin's enrichment, it has also been widely observed that centromeres split for considerable distances in pre-anaphase cells. In budding yeast, the region of splitting lies within the region of centromeric cohesin enrichment. Thus, it is conceivable that cohesin surrounding the separated sequences is important to restrain splitting and to maintain cohesion. However, these considerations do not solve the paradox of how centromere splitting can occur exactly at the place where cohesin is most highly enriched. We have now shown that cohesin disappears from sequences that undergo splitting. The region of cohesin removal, like the region of splitting, is confined within the region of centromeric cohesin enrichment. This provides a solution to how, at least in budding yeast, cohesin is enriched at centromeres while sequences within the region of enrichment separate in response to the bipolar tension produced during bi-orientation on the metaphase spindle.

\section{Cohesin removal during centromere splitting}

The observation that cohesin disappears from centromeres during breathing suggests that sister chromatid cohesion is lost at these regions. It does not explain how cohesin is lost from centromeres, and, in particular, whether loss of cohesin predates and facilitates, or is the consequence of, centromere breathing. Two observations suggest that the latter is the case and that disappearance of cohesin may be the result of the forceful physical separation of centromeres under the influence of the mitotic spindle. First, we found that increasing the extent of centromere splitting by Cdc14mediated spindle stabilisation further decreased the amount of cohesin at centromeres. This suggests that cohesin removal responds to the actual separation of sister centromeres. Furthermore, cohesin that was loaded onto chromosomes before DNA replication, and that was therefore likely involved in promoting sister chromatid cohesion, disappeared completely from centromeres during breathing. In contrast, post-replicatively loaded, and therefore noncohesive, cohesin remained associated with centromeres. Considering the likely topological interaction of cohesin with chromosomes (Ivanov and Nasmyth 2005), we imagine that the cohesive embrace of sister chromatids is disrupted by the spindle force. This force only acts on cohesin that is actually involved in linking sister chromatids, but not on post-replicatively loaded cohesin that may embrace or associate with only one of the two sisters. This interpretation also supports the idea that single cohesin rings embrace both sister chromatids. Splitting of the sisters trapped within one cohesin ring would inevitably lead to breakage of the ring. If cohesion between sister chromatids was mediated by an interaction between two cohesin complexes on both sisters, their forceful separation might result in disruption of the inter-cohesin interaction, while individual cohesin rings could remain associated with their respective chromatids.

Cohesin has the ability to translocate along chromosomes, downstream of expressed genes, in response to their transcriptional activation (Glynn et al. 2004; Lengronne et al. 2004). Therefore, we considered the possibility that cohesin may not be removed from centromeres, but may slide away laterally towards surrounding sequences during breathing. However, we could not detect any increase in cohesin association next to where cohesin was lost at the centromere. This suggests that rather than sliding away to the sides, cohesin loses its contact with DNA during removal by centromere breathing. Loss of sister chromatid cohesion in response to mitotic spindle attachment has been observed between small minichromosomes containing core centromeric sequences (Tanaka et al. 1999). This is consistent with the idea that at least a certain number of cohesin molecules that link sister centromeres can indeed be disrupted by spindle force.

If the spindle force is sufficiently strong to disrupt or remove cohesin, how is the extent of centromere splitting and cohesin removal contained during centromere breathing? Centromeres have been observed to split for up to $0.8 \mu \mathrm{m}$, which spans much, but not all, of the length of the metaphase spindle. Furthermore, cohesin cleavage in metaphase is sufficient to trigger elongation of the spindle and separation of sister centromeres far into opposite daughter cells (Uhlmann et al. 2000). This suggests that cohesin limits the extent of centromere splitting. As centromeres move apart during splitting, non-cohesive centromeric chromatin is exposed that links the point of 
spindle attachment at the core centromere to the surrounding cohesin-bound regions. These sequences have been observed to undergo significant elastic stretch ( $\mathrm{He}$ et al. 2000). The spring-like behaviour of the centromeric chromatin will consequently reduce the impact of microtubule forces on the surrounding sites of sister chromatid cohesion such that cohesin integrity may be protected once the spring reaches sufficient length and therefore elasticity.

The region of cohesin removal displayed apparently sharp boundaries. This was pronounced at several of the centromeres that contained both pre- and post-replicatively loaded cohesin at the time of their analysis. Closer inspection revealed that these boundaries often coincided with sites of convergent transcriptional termination. Therefore, it could be that the sharpness of the boundaries is the consequence of the positioning of remaining non-cohesive cohesin by the transcriptional machinery rather than a reflection of cohesin removal. In particular, this could be the case where the boundaries appear to be placed asymmetrically around centromeres, a situation that is difficult to explain by cohesin removal which is expected to progress symmetrically from the core centromeric site of microtubule attachment. When only post-replicatively loaded cohesin at the centromere of chromosome 6 was analysed, a more gradual response of removal with the distance from the core centromere was observed, consistent with a population of centromeres in a range of conformations.

\section{Cohesion re-establishment during centromere re-association}

Cohesin removal from centromeres during breathing does not appear to be an irreversible event. We find that cohesin re-accumulates, and centromeric sister chromatid cohesion is likely re-established during centromere re-association after spindle disruption. Sister centromere re-association after splitting, or at least a reduction of their distance of splitting, is frequently observed during undisturbed mitotic progression (He et al. 2000; Tanaka et al. 2000; Goshima and Yanagida 2001; Pearson et al. 2001). We speculate that the same process of cohesin re-accumulation and cohesion re-establishment occurs in a dynamic fashion during this breathing behaviour of centromeres in the normal chromosome bi-orientation process. Bi-orientation is based on tension as a balance of the spindle pulling force and a counter-acting force between sister centromeres. Therefore, it is possible that centromere re-association and re-establishment of sister chromatid cohesion forms part of the chromosomal counterforce.

In principle, stable non-elastic cohesion between sister centromeres could provide the necessary counterforce to microtubule attachment. However, there might be several reasons for why a more dynamic behaviour of centromeric sequences is observed. First of all, cohesin-mediated sister chromatid cohesion may simply not be strong enough to resist the full force of the mitotic spindle. While this may appear counter-intuitive, a finite strength of cohesin may be advantageous. Chromosomal cohesin cleavage by separase in anaphase is an efficient process (Hornig and Uhlmann 2004), but it is hard to exclude that on occasion a small number of cohesin rings remain intact. These uncleaved complexes, if not too stable, could be disrupted during chromosome segregation, instead of causing DNA breaks or segregation defects of otherwise correctly separating sister chromatids. In this scenario, centromere breathing is the mere consequence of limited cohesin stability. This would not predict, or require, that centromere cohesion is re-established during breathing. A second, not mutually exclusive, possibility is that centromere breathing is the consequence of intrinsic mitotic microtubule dynamics. Despite the overall pulling force exerted by the spindle, individual microtubules are in a dynamic equilibrium of growth and shrinking. This dynamic instability is thought to aid the search and capture mechanism of kinetochore attachment, as well as the error correction process of faulty attachments. Although microtubules are thought to stabilise after reaching productive kinetochore interactions, an elastic response of centromeric sequences to attachment will facilitate continuous interactions with dynamic microtubules. Both microtubule-driven poleward and antipoleward kinetochore movements in metaphase have been documented in newt lung cells (Skibbens et al. 1993). In this model, centromere breathing reflects movements dictated by microtubule dynamics. Transitions between poleward and anti-poleward movement are likely influenced by tension that builds up during splitting. Therefore, re-establishment of sister chromatid cohesion would ensure that the counterforce during repeated cycles of poleward and anti-poleward movement remains intact. A third possibility is that centromere splitting during chromosome bi-orientation serves an important regulatory role. The aurora B kinase complex promotes the turnover of microtubules, including frequent detachment from kinetochores, until bipolar attachment is reached. Aurora B kinase resides between sister centromeres, and it has been proposed that microtubule-kinetochore interactions are stabilised as centromeres move away from the kinase complex during breathing (Tanaka et al. 2002; Dewar et al. 2004). In this model, centromere splitting is required to stabilise microtubule attachment under tension. Should attachment be inadvertently lost after establishment of tension, centromere re-association would be required to reset the tension sensor to allow the bi-orientation process to restart.

In the absence of the cohesion establishment factor Eco1, re-association of sister centromeres after spindle disruption was significantly reduced. This suggests that re-establishment of sister chromatid cohesion contributes to centromere 
re-association. The mechanism by which cohesion might be re-established at centromeres outside the normal period of cohesion establishment in S-phase is unknown. The observation that Ecol is required suggests that the role of Ecol may not be exclusively linked to DNA replication. Rather, Eco1 may function as a more general factor that promotes cohesin ring opening or its turnover on DNA. This activity may normally be harnessed for cohesion establishment in Sphase by Eco1 recruitment to the replication fork via its interaction with PCNA (Lengronne et al. 2006; Moldovan et al. 2006). In mitosis, Ecol may facilitate cohesive reassociation of cohesin with sister centromeres, while postreplicatively loaded cohesin along chromosome arms does not establish sister chromatid cohesion. It will be interesting to determine if Ecol is present at mitotic centromeres to fulfil this role. We do not know whether cohesion reestablishment at centromeres is an active process that in fact provides a force towards centromere re-association. If not, cohesin might establish linkages between sister sequences that regain contact with each other by diffusion or another directed mechanism. Ecol function is essential for cohesion establishment during S-phase, but becomes dispensable for cell survival after DNA replication (Skibbens et al. 1999; Tóth et al. 1999). This suggests that either centromere breathing is not an essential aspect of mitosis or that mechanisms in addition to cohesion re-establishment contribute to centromere elasticity. Mutation of Ecol reduces the efficiency of centromere re-association, but does not eliminate it. Additional mechanisms that promote sister centromere re-association might include anti-poleward centromere movement driven by growing kinetochore microtubules and recoiling of elastic chromatin deformations that are observed during breathing (Skibbens et al. 1993; He et al. 2000).

Taken together, we have provided observations that can explain the centromere breathing paradox within our current knowledge about the cohesin complex. At the same time, these observations raise new questions about the mechanism of mitosis-specific cohesin loading at centromeres and the apparent re-establishment of sister chromatid cohesion at this locus outside S-phase.

Acknowledgements We are grateful to T. Rolef Ben-Shahar and J. Mc Intyre for valuable help. We thank T. Toda and all members of our laboratory for insightful discussions and critical reading of the manuscript. This work was supported by the Human Frontier Science Program. This material is based upon work supported by the National Science Foundation under a grant awarded in 2006 to M.T.O.H.

\section{References}

Bernard P, Maure JF, Partridge JF, Genier S, Javerzat JP, Allshire RC (2001) Requirement of heterochromatin for cohesion at centromeres. Science 294:2539-2542
Blat Y, Kleckner N (1999) Cohesins bind to preferential sites along yeast chromosome III, with differential regulation along arms versus the centric region. Cell 98:249-259

Ciosk R, Shirayama M, Shevchenko A, Tanaka T, Toth A, Shevchenko A, Nasmyth K (2000) Cohesin's binding to chromosomes depends on a separate complex consisting of Scc2 and Scc4 proteins. Mol Cell 5:1-20

Dewar H, Tanaka K, Nasmyth K, Tanaka TU (2004) Tension between two kinetochores suffices for their bi-orientation on the mitotic spindle. Nature 428:93-97

Eckert CA, Gravdahl DJ, Megee PC (2007) The enhancement of pericentromeric cohesin association by conserved kinetochore components promotes high-fidelity chromosome segregation and is sensitive to microtubule-based tension. Genes Dev 21: 278-291

Glynn EF, Megee PC, Yu HG, Mistrot C, Unal E, Koshland DE, DeRisi JL, Gerton JL (2004) Genome-wide mapping of the cohesin complex in the yeast Saccharomyces cerevisiae. PLoS Biol 2:1325-1339

Goshima G, Yanagida M (2000) Establishing biorientation occurs with precocious separation of the sister kinetochores, but not the arms, in the early spindle of budding yeast. Cell 100:619-633

Goshima G, Yanagida M (2001) Time course analysis of precocious separation of sister centromeres in budding yeast: continuously separated or frequently reassociated? Genes Cells 6:765-773

Haering CH, Schoffnegger D, Nishino T, Helmhart W, Nasmyth K, Löwe J (2004) Structure and stability of cohesin's Smc1-kleisin interaction. Mol Cell 15:951-964

He X, Asthana S, Sorger PK (2000) Transient sister chromatid separation and elastic deformation of chromosomes during mitosis in budding yeast. Cell 101:763-775

Higuchi T, Uhlmann F (2005) Stabilisation of microtubule dynamics at anaphase onset promotes chromosome segregation. Nature 433:171-176

Hirano T (2006) At the heart of the chromosome: SMC proteins in action. Nat Rev Mol Cell Biol 7:311-322

Hornig NCD, Uhlmann F (2004) Preferential cleavage of chromatinbound cohesin after targeted phosphorylation by Polo-like kinase. EMBO J 23:3144-3153

Hughes-Schrader S (1950) The "pre-metaphase stretch" and kinetochore orientation in phasmids. Chromosoma 3:1-21

Ivanov D, Nasmyth K (2005) A topological interaction between cohesin rings and a circular minichromosome. Cell 122:849 860

Katou Y, Kanoh Y, Bandoh M, Noguchi H, Tanaka H, Ashikari T, Sugimoto K, Shirahige K (2003) S-phase checkpoint proteins Tof1 and Mrc1 form a stable replication-pausing complex. Nature 424:1078-1083

Kitajima TS, Hauf S, Ohsugi M, Yamamoto T, Watanabe Y (2005) Human Bub1 defines the persistent cohesion site along the mitotic chromosome by affecting shugoshin localization. Curr Biol 15:353-359

Knop M, Siegers K, Pereira G, Zachariae W, Winsor B, Nasmyth K, Schiebel E (1999) Epitope tagging of yeast genes using a PCRbased strategy: more tags and improved practical routines. Yeast 15:963-972

Lengronne A, Katou Y, Mori S, Yokobayashi S, Kelly GP, Itoh T, Watanabe Y, Shirahige K, Uhlmann F (2004) Cohesin relocation from sites of chromosomal loading to places of convergent transcription. Nature 430:573-578

Lengronne A, McIntyre J, Katou Y, Kanoh Y, Hopfner K-P, Shirahige K, Uhlmann F (2006) Establishment of sister chromatid cohesion at the S. cerevisiae replication fork. Mol Cell 23:787-799

Losada A, Hirano M, Hirano T (1998) Identification of Xenopus SMC protein complexes required for sister chromatid cohesion. Genes Dev 12:1986-1997 
Losada A, Yokochi T, Kobayashi R, Hirano T (2000) Identification and characterization of $\mathrm{SA} / \mathrm{Scc} 3 \mathrm{p}$ subunits in the Xenopus and human cohesin complexes. J Cell Biol 150:405-416

McGuinness BE, Hirota T, Kudo NR, Peters J-M, Nasmyth K (2005) Shugoshin prevents dissociation of cohesin from centromeres during mitosis in vertebrate cells. PLoS Biol 3:433-449

Megee PC, Mistrot C, Guacci V, Koshland D (1999) The centromeric sister chromatid cohesion site directs Mcd1p binding to adjacent sequences. Mol Cell 4:445-450

Michaelis C, Ciosk R, Nasmyth K (1997) Cohesins: chromosomal proteins that prevent premature separation of sister chromatids. Cell 91:35-45

Moldovan G-L, Pfander B, Jentsch S (2006) PCNA controls establishment of sister chromatid cohesion during $\mathrm{S}$ phase. Mol Cell 23:723-732

Nabeshima K, Nakagawa T, Straight AF, Murray A, Chikashige Y, Yamashita YM, Hirakoa Y, Yanagida M (1998) Dynamics of centromeres during metaphase-anaphase transition in fission yeast: Dis1 is implicated in force balance in metaphase bipolar spindle. Mol Biol Cell 9:3211-3225

Nasmyth K, Haering CH (2005) The structure and function of SMC and kleisin complexes. Ann Rev Biochem 74:595-648

Nasmyth K, Schleifer A (2004) From a single double helix to paired double helices and back. Philos Trans R Soc B 359:99-108

Nonaka N, Kitajima T, Yokobayashi S, Xiao G, Yamamoto M, Grewal SIS, Watanabe Y (2002) Recruitment of cohesin to heterochromatic regions by Swi6/HP1 in fission yeast. Nat Cell Biol 4:89-93

Pearson CG, Maddox PS, Salmon ED, Bloom K (2001) Budding yeast chromosome structure and dynamics during mitosis. J Cell Biol 152:1255-1266

Salic A, Waters JC, Mitchison TJ (2004) Vertebrate shugoshin links sister centromere cohesion and kinetochore microtubule stability in mitosis. Cell 118:567-578

Shelby RD, Hahn KM, Sullivan KF (1996) Dynamic elastic behavior of a-satellite DNA domains visualized in situ in living human cells. J Cell Biol 135:545-557

Sjögren C, Nasmyth K (2001) Sister chromatid cohesion is required for postreplicative double-strand break repair in Saccharomyces cerevisiae. Curr Biol 11:991-995

Skibbens RV, Skeen VP, Salmon ED (1993) Directional instability of kinetochore motility during chromosome congression and segre- gation in mitotic newt lung cells: a push-pull mechanism. J Cell Biol 122:859-875

Skibbens RV, Corson LB, Koshland D, Hieter P (1999) Ctf7p is essential for sister chromatid cohesion and links mitotic chromosome structure to the DNA replication machinery. Genes Dev 13:307-319

Ström L, Lindroos HB, Shirahige K, Sjögren C (2004) Postreplicative recruitment of cohesin to double-strand breaks is required for DNA repair. Mol Cell 16:1003-1015

Tanaka T, Cosma MP, Wirth K, Nasmyth K (1999) Identification of cohesin association sites at centromeres and along chromosome arms. Cell 98:847-858

Tanaka T, Fuchs J, Loidl J, Nasmyth K (2000) Cohesin ensures bipolar attachment of microtubules to sister centromeres and resists their precocious separation. Nat Cell Biol 2:492-499

Tanaka TU, Rachidi N, Janke C, Pereira G, Galova M, Schiebel E, Stark MJR, Nasmyth K (2002) Evidence that the Ipl1-Sli15 (aurora kinase-INCENP) complex promotes chromosome biorientation by altering kinetochore-spindle pole connections. Cell 108:317-329

Tóth A, Ciosk R, Uhlmann F, Galova M, Schleiffer A, Nasmyth K (1999) Yeast cohesin complex requires a conserved protein, Ecolp (Ctf7), to establish cohesion between sister chromatids during DNA replication. Genes Dev 13:320-333

Uhlmann F (2003) Chromosome cohesion and separation: from men and molecules. Curr Biol 13:R104-R114

Uhlmann F, Nasmyth K (1998) Cohesion between sister chromatids must be established during DNA replication. Curr Biol 8:1095-1101

Uhlmann F, Wernic D, Poupart M-A, Koonin EV, Nasmyth K (2000) Cleavage of cohesin by the CD clan protease separin triggers anaphase in yeast. Cell 103:375-386

Waizenegger IC, Hauf S, Meinke A, Peters J-M (2000) Two distinct pathways remove mammalian cohesin complexes from chromosome arms in prophase and from centromeres in anaphase. Cell 103:399-410

Warren WD, Steffensen S, Lin E, Coelho P, Loupart M-L, Cobbe N, Lee JY, McKay MJ, Orr-Weaver T, Heck MMS, Sunkel CE (2000) The Drosophila RAD21 cohesin persists at the centromere region in mitosis. Curr Biol 10:1463-1466

Weber SA, Gerton JL, Polancic JE, DeRisi JL, Koshland D, Megee PC (2004) The kinetochore is an enhancer of pericentric cohesin binding. PLoS Biol 2:1340-1353 